\title{
Long non-coding RNA SNHG1 regulates NOB1 expression by sponging miR-326 and promotes tumorigenesis in osteosarcoma
}

\author{
JIANDONG WANG, LEI CAO, JIANHONG WU and QIUGEN WANG
}

\author{
Department of Trauma and Orthopedics, Trauma Emergency Center, \\ Shanghai General Hospital, Shanghai Jiao Tong University, Shanghai 200080, P.R. China
}

Received May 27, 2017; Accepted October 8, 2017

DOI: $10.3892 /$ ijo.2017.4187

\begin{abstract}
The long non-coding RNA (lncRNA) small nucleolar RNA host gene 1 (SNHG1) has been demonstrated to participate in the deterioration of many types of cancer. However, the underlying mechanisms of SNHG1-mediating functions in osteosarcoma (OS) have yet to be elucidated. In the present study, our results showed that SNHG1 was upregulated in OS tissues and cell lines, and high SNHG1 expression predicts poor overall survival of OS patients. Knockdown of SNHG1 inhibited cell growth and metastasis of OS in vitro and in vivo. Furthermore, our data demonstrated that there was reciprocal repression between SNHG1 and miR-326 which act as a tumor suppressor in OS cells, and exhibiting a strong negative relationship between SNHG1 and miR-326 expression in OS tissues. Additionally, we identified that SNHG1 increased human nin one binding protein (NOB1), an oncogene, through sponging miR-326 as competing endogenous RNA (ceRNA), finally prompting cell growth, migration and invasion in OS. Collectively, these findings not only uncovered that the SNHG1/miR-326/NOB1 signaling axis has a key role in OS progression but also suggested the potential application of SNHG1 and miR-326 as biomarkers in the OS diagnosis and treatment.
\end{abstract}

\section{Introduction}

Osteosarcoma (OS), a primary malignant bone tumor, which is characterized by a highly malignant tendency to rapidly damage the surrounding tissues and to metastasize (1). The global incidence rate is estimated at 4 million per year, with a peak incidence in children and adolescents (2). Despite current therapeutic strategies including multi-agent chemotherapy,

Correspondence to: Dr Qiugen Wang, Department of Trauma and Orthopedics, Trauma Emergency Center, Shanghai General Hospital, Shanghai Jiao Tong University, No. 85 Wujin Road, Shanghai 200080, P.R. China

E-mail: wangqiugen@126.com

Key words: long non-coding RNA SNHG1, osteosarcoma, cell growth, metastasis, miR-326, human nin one binding protein surgery and radiotherapy, the survival of OS patients remains poor, since $\sim 80 \%$ of patients with surgical treatment eventually develop recurrent metastatic OS (3), with 5-year survival rate of only $50-60 \%$ (4). Therefore, it is essential to clarify the molecular mechanism underlying OS tumorigenesis and investigate the new molecular targets inhibiting tumor growth and metastasis in OS clinical diagnosis and treatment.

Increasing evidence have demonstrated that non-coding RNAs (ncRNAs) including microRNA (miRNA) and long non-coding RNA (lncRNA) have been well reported as a novel class of potential therapeutic targets and clinical biomarkers for cancers (5-7). miRNAs are small ncRNAs averaging 20-24 nucleotides, which modulate translational suppression or mRNA degradation by binding to their 3'-untranslated region (3'-UTR) (8). Additionally, lncRNAs, are defined as ncRNAs more than 200 nucleotides in length, have been identified to have key regulatory roles via regulating gene expression, such as intergenic regions, overlapping, antisense and intronic to protein-coding genes (9-11). The growing number of evidence demonstrated that lncRNAs have an important role in tumor biology, including tumor initiation, progression and metastasis $(12,13)$. IncRNAs aberrant expressions have been confirmed in different cancers, such as hepatocellular carcinoma, gastric, lung and cervical cancer $(10,11,14,15)$. In OS, some lncRNAs were confirmed to act as tumor suppressors (MEG3, HIF2PUT and TUSC7) or exhibit oncogenic properties (H19, MALAT1, ANCR, HOTAIR and TUG1) to control OS progression by modulating cell cycle or apoptosis to regulate cell proliferation or migration 16). Tumor suppressor candidate 7 (TUSC7), a potential tumor suppressor, which was remarkably downregulated in OS tissues, and low TUSC7 expression is associated with poor survival in OS patients (17). Li et al (18) confirmed that lncRNA HOPTTIP was overexpressed in OS tissues, and was correlated with advanced clinical stage and distant metastasis. Inhibition of HOPTTIP in OS cells dramatically repressed cell proliferation, migration and invasion. Therefore, the biological role of lncRNAs were further investigated in OS to develop a novel prognostic marker and potential therapeutic target in OS patients.

It is well documented that many miRNAs have been identified to act as tumor suppressor genes or oncogenes in cancers and could block cell signaling pathways to suppress different biological processes, such as cell proliferation, apoptosis, differentiation and migration $(19,20)$. Recently, lncRNA was 
identified to be a sponge for mediating the miRNA expression and activity as competing endogenous RNA (ceRNA) (21-23). A recent study demonstrated that IncRNA small nucleolar RNA host gene 1 (SNHG1) was upregulated in hepatocellular carcinoma (HCC) tissues and cells, and promoted HCC cells proliferation and cycle progression (24). Growing evidence uncovered that ectopic expression of SNHG1 could function as oncogenes in various cancers including lung and breast cancer (24-26). However, the roles of SNHG1 in OS have not yet been elucidated. Moreover, increasing evidence confirmed that SNHG1 acts as crucial regulator to modulate various cancer progressions through competitively binding miRNAs including miR-199a-3p and miR-195 $(27,28)$. These inspired us to explore the roles of SNHG1 in OS and investigate the relationship between SNHG1 and miRNAs in the carcinogenesis of OS.

In the present study, our results showed that SNHG1 was upregulated in OS tissues and cell lines, knockdown of SNHG1 suppressed cell growth and metastasis in vitro and in vivo. Subsequently, we explored the molecular mechanism of SNHG1 in OS progression and the reciprocal regulation between SNHG1 and miR-326. Our findings uncovered that the SNHG1/miR-326/human nin one binding protein (NOB1) signaling pathway play an important role in OS tumorigenesis and suggested the potential value of SNHG1 in OS clinical diagnosis and treatment.

\section{Materials and methods}

Patient tissue samples and OS cell lines. Forty-five paired tissue samples of OS and adjacent normal tissues were obtained from patients in the Department of Trauma and Orthopedics, Shanghai General Hospital, Shanghai Jiao Tong University. These patients did not receive preoperative radio therapy or chemotherapy before surgical resection. Histological diagnosis and differentiation were assessed independently by three pathologists according to the WHO classification system (29). Fresh specimens were stored at $-70^{\circ} \mathrm{C}$ until use. All patients agreed to provide their tumor tissues for clinical research through signing written informed consent. The Institute Research Medical Ethics Committee of China Medical University approved the study protocol. The human OS cell lines MG-63, U2OS, Saos-2 and SOSP-9607 were purchased from the the American Type Culture Collection (ATCC; Rockville, MD, USA) and grown in RPMI-1640 medium supplemented with $10 \%$ fetal bovine serum (FBS; Sigma-Aldrich, St. Louis, MO, USA), $100 \mathrm{IU} / \mathrm{ml}$ penicillin and $100 \mathrm{mg} / \mathrm{ml}$ streptomycin, at $37^{\circ} \mathrm{C}$ in a humidified atmosphere containing $5 \% \mathrm{CO}_{2}$. The osteoblastic cell line hFOB 1.19 was maintained in Dulbecco's modified Eagle's minimal essential medium (DMEM)/F12; Gibco, Carlsbad, CA, USA) supplemented with $10 \% \mathrm{FBS}, 100 \mathrm{IU} / \mathrm{ml}$ penicillin and $100 \mathrm{mg} / \mathrm{ml}$ streptomycin.

Quantitative real-time PCR. Total RNA was extracted from frozen tissues and cell samples with TRIzol reagent (Invitrogen, Carlsbad, CA, USA) according to the manufacturer's instructions. High Capacity cDNA Reverse Transcription kit and TaqMan MicroRNA Reverse Transcription kit were applied to IncRNAs and miRNA reverse transcription, respectively
(Applied Biosystems, Foster City, CA, USA). Quantitative RT-PCR was performed by TaqMan Universal Master Mix II with TaqMan non-coding RNA assays of SNHG1 and GAPDH or with TaqMan microRNA assays of miR-326 and U6 (Applied Biosystems). GAPDH and U6 were used as internal controls. The relative expression levels were determined using the $2^{-\Delta \Delta \mathrm{Ct}}$ method.

Oligonucleotide and plasmid transfection. Small interfering RNA si-SNHG1 (5'-GAG AGC TCT GTT GTT GCA ATG TTCA-3') and scrambled negative control si-Scramble (5'-GAG TCT CGT TGC GTT GTA ATG ATCA-3') oligonucleotides were obtained from Shanghai GenePharma Co., Ltd. (Shanghai, China). The miR-326 mimics or inhibitor and corresponding negative control (NC) were obtained from Guangzhou RiboBio Co., Ltd. (Guangzhou, China). To overexpress SNHG1, pcDNASNHG1 was amplified by PCR, and the final product was inserted into the pcDNA3.1 plasmid (Invitrogen). SNHG1 mutants were performed by the QuikChange Site-Directed Mutagenesis kit (Stratagene, La Jolla, CA, USA) according to the manufacturer's instructions. The OS cells were seeded into each well of a 6-well plate, cultured in the appropriate culture media containing $10 \%$ FBS. Then, the cells were transfected with si-SNHG1, si-Scramble, miR-326 mimics, miR-326 inhibitor, pcDNA-SNHG1 and pcDNA-mut-SNHG1. Cells were collected $48 \mathrm{~h}$ after transfection.

Cell Counting kit- 8 assay. The proliferation of cells was determined by the Cell Counting kit- 8 (CCK-8) assay according to the manufacturer's instructions. After transfection, the OS cells $\left(5 \times 10^{4}\right.$ cells/well) were seeded in 96 -well plate with $100 \mu \mathrm{l}$ DMEM medium supplemented with $10 \%$ FBS. After 48-h incubation, CCK- 8 reagent $(10 \mu \mathrm{l})$ was added to each well and continuously cultured for $1 \mathrm{~h}$ in $5 \% \mathrm{CO}_{2}$ (Thermo Fisher Scientific, Waltham, MA, USA). The absorbance rate at $450 \mathrm{~nm}$ was measured by microplate reader (Bio-Rad Laboratories, Hercules, CA, USA). All experiments were performed in quintuplicate on three separate occasions.

Analysis of apoptosis. Approximately $48 \mathrm{~h}$ after transfection, $1 \times 10^{6}$ cells were collected and washed twice with HEPESbuffered saline. After treatment with trypsin, cells were fixed with $70 \%$ ice-cold methanol at $4^{\circ} \mathrm{C}$ for $30 \mathrm{~min}$. Cells were then resuspended in binding buffer and stained with $5 \mu \mathrm{l}$ of Annexin V-FITC (BD Biosciences, Mountain View, CA, USA) and $1 \mu \mathrm{l}$ of propidium iodide (PI, $50 \mu \mathrm{g} / \mathrm{ml}$ ) (BD Biosciences). Flow cytometric evaluation was performed within $5 \mathrm{~min}$. Stained cells were measured by flow cytometry (FACSCalibur; BD Biosciences). The measurements were performed independently for at least three times with similar results.

Western blot analysis. OS cells were treated with ice-cold whole cell extraction buffer ( $\mathrm{pH}$ 7.6) combing the protease inhibitors [0.5 mM DTT, $20 \mathrm{mM}$ HEPES, $0.2 \mathrm{mM}$ EDTA, $75 \mathrm{mM} \mathrm{NaCl}$, $2.5 \mathrm{mM} \mathrm{MgCl}{ }_{2}, 0.1 \%$ Triton X-100, $50 \mathrm{mM} \mathrm{NaF}, 0.1 \mathrm{mM}$ $\mathrm{Na}_{3} \mathrm{VO}_{4}$, and the protease inhibitors including $1 \mathrm{mg} / \mathrm{ml}$ aprotinin, $0.5 \mathrm{mg} / \mathrm{ml}$ leupeptin and $100 \mathrm{mg} / \mathrm{ml} \mathrm{4-(2-aminoethyl)}$ benzenesulfonyl fluoride]. The BCA protein assay kit (Pierce, Rockford, IL, USA) was used to measure protein concentration. Equal amounts of protein samples $(60 \mathrm{mg})$ were separated 
in $10 \%$ SDS-polyacrylamide gels (Sigma-Aldrich) and then transferred onto polyvinylidene difluoride (PVDF) membranes (BD Biosciences, San Diego, CA, USA). The membranes were blocked with $5 \%$ skim milk at room temperature for $1 \mathrm{~h}$ and incubated with primary antibodies at $4^{\circ} \mathrm{C}$ overnight against cle-caspase-3, ZEB1, vimentin, N-Cadherin and NOB1, which were purchased from Santa Cruz Biotechnology (Santa Cruz, CA, USA). The goat anti-mouse IgG horseradish peoxidase antibodies were obtained from Santa Cruz Biotechnology. The bands were scanned using the ChemiDoc XRS + Imaging System (Bio-Rad Laboratories) and quantified using Quantity One v4.6.2 software (Bio-Rad Laboratories).

Xenograft tumor model. All animal procedures were carried out with the protocols approved by the Animal Care Committee of the Shanghai General Hospital, Shanghai Jiao Tong University. The BALB/C athymic nude mice (4 weeks of age) were obtained from the Cancer Institute of the Chinese Academy of Medical Science. Mice were housed and maintained in laminar flow cabinets under specific pathogen free conditions with free access to food and water. When the female BALB/C athymic nude mice $(n=4)$ were $7-8$ weeks of age, each mouse was inoculated with Saos-2 or U2OS cells transfected with si-SNHG1 or si-Scramble in $0.2 \mathrm{ml}$ of medium subcutaneously in the forelimb. The tumor cells were allowed to grow for 4 weeks. The tumor growth was evaluated by the measurement of the length and the width with electronic calipers and the tumor volume was determined using the formula: Volume $\left(\mathrm{mm}^{3}\right)=\left(\right.$ length $\mathrm{x}$ width $\left.{ }^{2}\right) / 2$. The mice were sacrificed in 28 days, and then the tumors were excised and weighed.

Cell migration assay. The wound healing assay was applied to test cell migration. The Saos-2 and U2OS cells were plated onto 6-well plates and transfected with si-SNHG1 or si-Scramble. The migration status was evaluated by measuring the movement of cells into a scraped area created by a micropipette pipette tip. The spread of wound closure was observed after $24 \mathrm{~h}$. The wound area was measured and the percentage of closure of denuded area was calculated using the ImageJ software (NIH, Bethesda, MD, USA).

Cell invasion assay. Cell invasion assays were conducted by 24-well Transwell chambers with $8.0 \mu \mathrm{m}$ pore size polycarbonate membrane (Corning Inc., Corning, NY, USA). The Saos-2 and U2OS cells were seeded on the top side of the membrane precoated with Matrigel (BD Biosciences, Franklin Lakes, NJ, USA) and then transfected with si-SNHG1 or si-Scramble. After $48 \mathrm{~h}$ of incubation, the non-invading cells were carefully removed from the upper surface and invading cells were fixed with methanol and stained with crystal violet (Sigma-Aldrich) on the lower surface of the membrane. The cell numbers were calculated in each well with six random fields at x200. Experiments were performed at least 3 times.

Luciferase assay. To construct reporter vector, one fragment of NOB1 3'-UTR [wild-type (wt) or mutant (mut), respectively] and two fragments of SNHG1 mRNA containing predicted miR-326 binding site 1 or 2 were separately amplified and fused to a modified pcDNA3.1 vector containing a luciferase gene, which was cloned into upstream of cloning sites. Mut reporter vectors were performed using the Mutagenesis kit (Stratagene). The OS cells U2OS were seeded into 24-well plates and transfected with the different reporter plasmid including pcDNA-wt-SNHG1-1, pcDNA-mut-SNHG1-1, pcDNA-wt-SNHG1-2, pcDNA-mut-SNHG1-2, pcDNAwt-NOB1 and pcDNA-mut-NOB1, together with miR-326 mimics/inhibitor or corresponding mimic/inhibitor $\mathrm{NC}$ using Lipofectamine 2000 (Invitrogen). Forty-eight hours after the transfection, the luciferase activity was determined by the Dual-Light luminescent reporter gene assay (Applied Biosystems). Each experiment was performed at least 3 times in individual experiments. The ratio of Renilla luciferase to firefly luciferase was calculated for each well.

Immunohistochemistry. Immunohistochemistry was used to detect NOB1 protein expression in OS tissues. NOB1 antibody was purchased from Santa Cruz Biotechnology. Immunohistochemistry was conducted on paraformaldehydefixed paraffin sections. The percentage of positive tumor cells was graded according to the following criteria: $0,<10 \% ; 1$, $10-30 \% ; 2,31-50 \% ; 3,>50 \%$. The OS tissues with different NOB1 expression from patients were divided into the lowexpression group (0 or 1) and the high-expression group (2 or 3$)$.

Statistical analysis. All statistical analyses were performed using SPSS 14.0 software (SPSS, Inc., Chicago, IL, USA). Experiments were conducted in triplicate and data are presented as the mean \pm SEM. The results were analyzed with the Student's t-test. The survival analysis was performed using the Kaplan-Meier method. $\mathrm{P}<0.05$ was defined as significant and $\mathrm{P}<0.01$ was defined very significant.

\section{Results}

lncRNA SNHGl is upregulated in OS tissues and cell lines. To determine lncRNAs involved in OS tumorigenesis, we screened GEO database and obtained one human lncRNA microarray datasets (https://www.ncbi.nlm.nih.gov/geo/query/ acc.cgi?acc $=$ GSE85537) to search differentially expressed lncRNAs between OS bone tissues and OS lung metastasis tissues. Especially, we observed that SNHG1 is upregulated in OS lung metastasis tissues compared with OS bone tissues in dataset (Fig. 1A). To validate this result, the quantitative reverse transcription-PCR (qRT-PCR) was used to examine SNHG1 expression in 44 pairs of tumor tissues and adjacent normal tissues. Our results showed that SNHG1 expression is significantly higher in tumor tissues than that in adjacent normal tissues $(\mathrm{P}<0.01$; Fig. 1B). Then, we measured SNHG1 levels in OS metastatic tissues $(n=24)$ and no-metastatic tissues $(n=20)$ using qRT-PCR. Consistent with OS tissue data, the SNHG1 expression levels were significantly upregulated in OS metastatic tissues compared with no-metastatic tissues $(\mathrm{P}<0.01$; Fig. 1C). To evaluate the correlation between SNHG1 expression and OS patients' prognosis, we performed the Kaplan-Meier survival analysis to plot OS curves and found that patients with high SNHG1 expression $(n=27)$ had a lower overall survival percentage than those with low SNHG1 expression $(n=17)(P<0.01$; Fig. 1D). Subsequently, the SNHG1 expression levels were further confirmed in OS cells, and the 
A
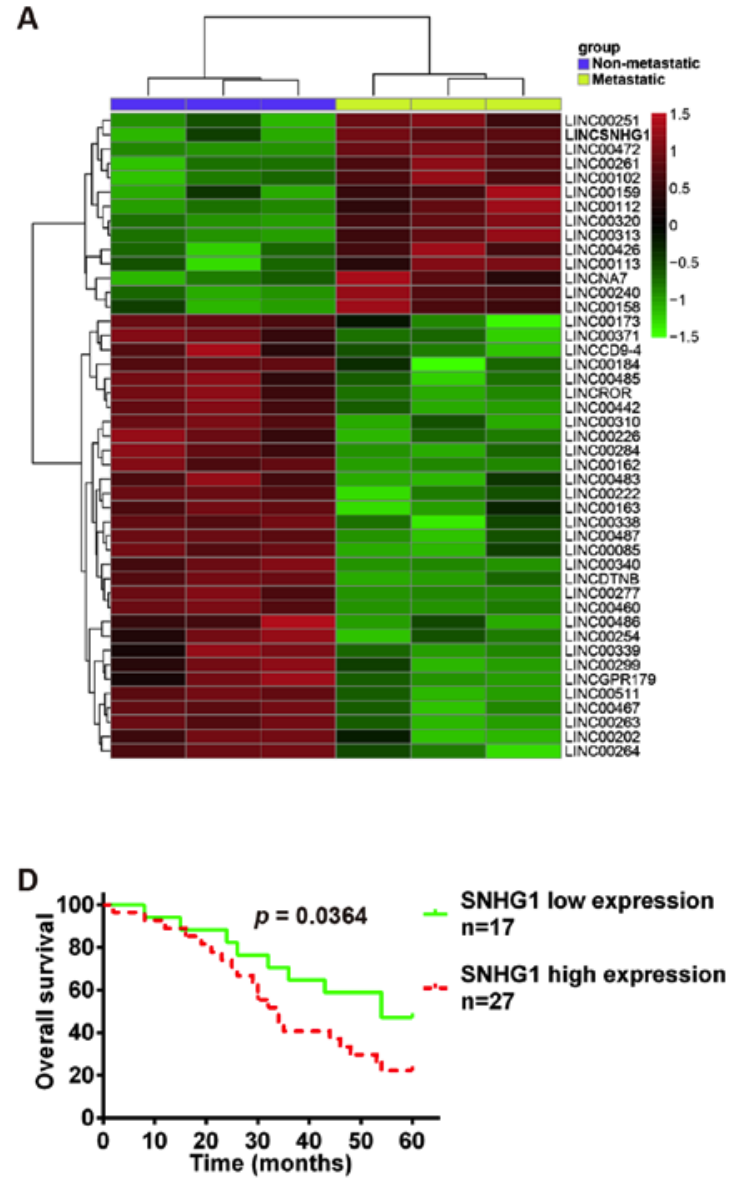

B

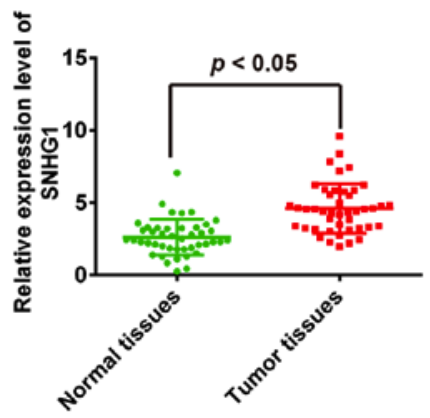

C

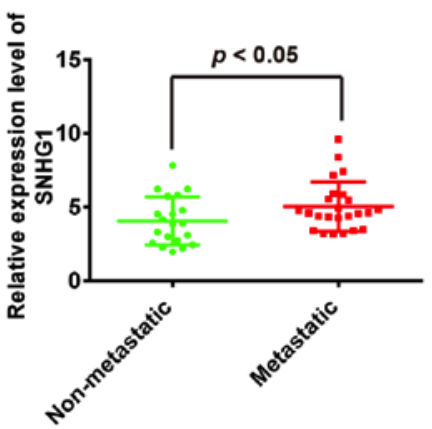

E

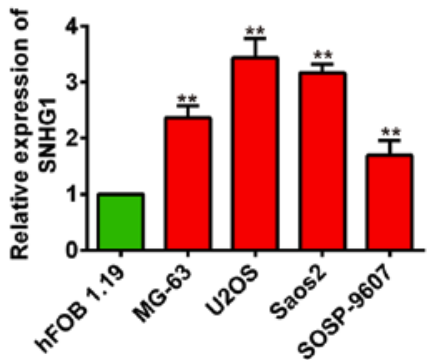

Figure 1. Expression levels of long non-coding RNA (lncRNA) SNHG1 in osteosarcoma (OS) tissues and matched normal tissues. (A) The microarray data obtained from the GEO database that showed differentially expressed lncRNAs between OS bone tissues and OS lung metastasis tissues (https://www. ncbi.nlm.nih.gov/geo/query/acc.cgi?acc=GSE85537). Red or green color in heatmap separately shows high or low expression, according to the color bar in logarithmic scale shown above the heatmap. (B) The levels of SNHG1 were quantified by qRT-TPCR analysis in tumor tissues and normal tissues ( $\mathrm{n}=44$ ). (C) The SNHG1 levels were measured in OS metastatic tissues $(n=24)$ and non-metastatic tissues $(n=20)$ using qRT-PCR. (D) Kaplan-Meier survival curves for OS patients with high SNHG1 expression ( $\mathrm{n}=27)$ or low SNHG1 expression $(\mathrm{n}=17)$. (E) The relative expression levels of SNHG1 were quantified by qRT-PCR in the four OS cell lines (MG-63, U2OS, Saos-2 and SOSP-9607) and human osteoblast cell line hFOB 1.19. Data are presented as the mean \pm SD of three independent experiments. ${ }^{* * *} \mathrm{P}<0.01$ vs. control.

SNHG1 expression is also significantly higher in OS cell lines MG-63, U2OS, Saos-2, SOSP-9607 than that in osteoblastic cell line hFOB 1.19 ( $\mathrm{P}<0.01$; Fig. 1E). Collectively, these data uncovered that SNHG1 expression is upregulated in OS tissues and high SNHG1 expression predicts poor prognosis in OS, suggesting SNHG1 might act as an oncogene in OS tumorigenesis.

Knockdown of SNHG1 inhibits OS cell proliferation, induces apoptosis and suppresses tumor growth. To explore the functions of SNHG1 in OS tumorigenesis, the Saos-2 and U2OS cells were transfected with si-SNHG1 and scrambled negative control si-Scramble. The results showed that SNHG1 level was markedly downregulated in Saos- 2 and U2OS cells transfected with si-SNHG1 compared with si-Scramble $(\mathrm{P}<0.01$; Fig. 2A and B). Subsequently, we performed the CCK- 8 assay to determine cell proliferation and its result showed that knockdown of SNHG1 significantly inhibited Saos-2 and U2OS cell proliferation compared with scrambled negative control si-Scramble ( $\mathrm{P}<0.01$; Fig. $2 \mathrm{C}$ and $\mathrm{D})$. Then, flow cytometric analysis was used to determine whether the effect of SNHG1 on OS cell proliferation is regulated by altering cell apoptosis. The Saos-2 and U2OS cells were stained with Annexin V-FITC and PI following transfection of cells with si-SNHG1 and si-Scramble. As shown in Fig. 2E, the apoptosis rates of Saos-2 and U2OS cells were increased in si-SNHG1 group compared with si-Scramble group $(\mathrm{P}<0.01)$. To further investigate the induction of apoptotic pathways following knockdown of SNHG1 in OS cells, the protein level of cleaved-caspase-3 was analyzed by western blot assays. Our results demonstrated that knockdown of SNHG1 significantly increased cleaved-caspase-3 expression level in si-SNHG1 group compared with si-Scramble group $(\mathrm{P}<0.01$; Fig. 2F). To investigate whether knockdown of SNHG1 affects tumorigenesis in vivo xenograft model was used, SNHG1-downregulating Saos-2 and U2OS cells and their parallel si-Scramble carrying cells were subcutaneously injected into the nude mice. The tumor growth was evaluated by measurement of tumor size and weight after injection. We observed that SNHG1-downregulating cells displayed significant decrease in the tumor size and weight compared with control groups $(\mathrm{P}<0.01$; Fig. $2 \mathrm{G}$ and $\mathrm{H})$. These data indicate 

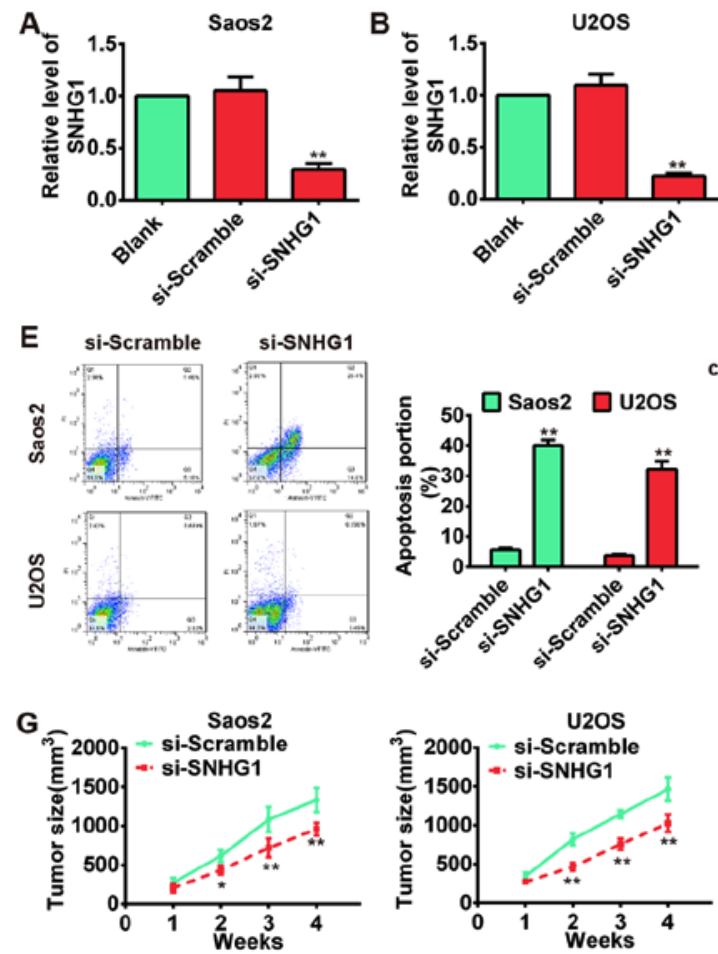
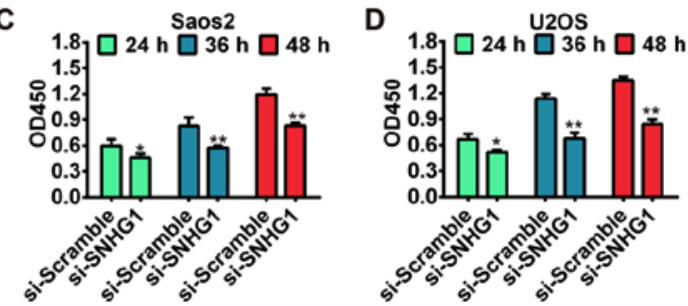

$\mathbf{F}$
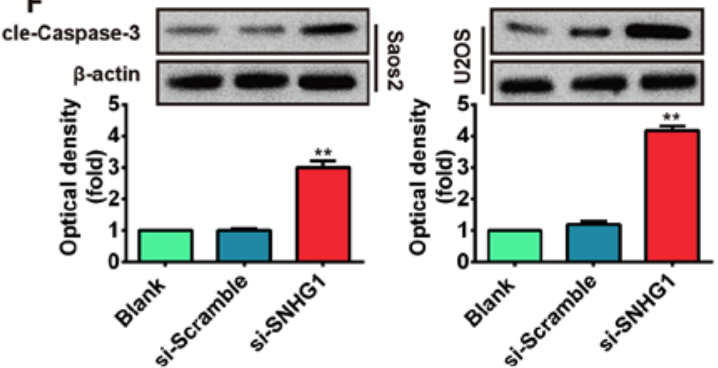

$\mathrm{H}$
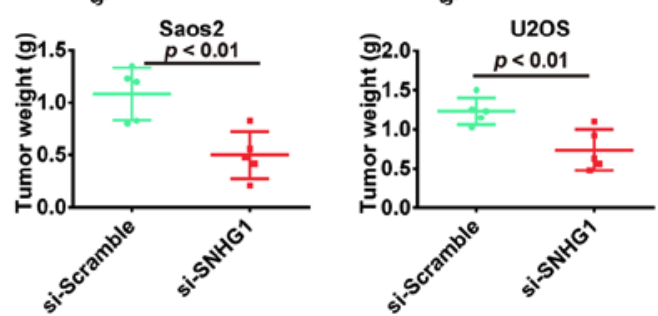

Figure 2. Effects of knockdown of SNHG1 on OS cells proliferation, apoptosis and tumor growth. (A and B) The interfering effects of si-SNHG1 were evaluated in Saos-2 and U2OS cells after transfection with si-SNHG1 or si-Scramble. (C and D) The Saos-2 and U2OS cells were transfected with si-SNHG1 or si-Scramble and the Cell Counting kit-8 (CCK-8) assay was used to detect the cell proliferation. (E and F) After transfection, the flow cytometric analysis and western blot analysis were performed to analyze cell apoptosis and cleaved-caspase- 3 expression, respectively. $\beta$-actin was used as an internal control for protein loading. (G and $\mathrm{H}$ ) The OS mouse model was established by using Saos-2 and U2OS cells transfected with si-SNHG1 or si-Scramble. The tumor size and weight were assessed in these two groups. The representative data were shown from one of three independent experiments with similar results. Data are presented as the mean \pm SD of three independent experiments. ${ }^{* *} \mathrm{P}<0.01,{ }^{*} \mathrm{P}<0.05$ vs. control.

that downregulation of SNHG1 possessed tumor-suppressive effects that could suppress cell proliferation and induce apoptosis in vitro and inhibit tumor growth in vivo.

Knockdown of SNHG1 inhibits cell migration and invasion in vitro. We further assessed the effects of SNHG1 downregulation on cell migration and invasion, which are key determinants of malignant progression and metastasis. We used wound healing assay to assess cell migration after transfection with si-SNHG1 or si-Scramble. As shown in Fig. 3A, the migration of Saos-2 and U2OS cells that knockdown of SNHG1 were significantly suppressed compared to the si-Scramble group $(\mathrm{P}<0.01)$. Subsequently, the transwell invasion assay was used to evaluate the cell invasion. We observed that knockdown of SNHG1 also markedly inhibited cell invasion in Saos-2 and U2OS cells after transfection with si-SNHG1 compared with that of si-Scramble $(\mathrm{P}<0.01$; Fig. 3B). These data demonstrated that knockdown of SNHG1 inhibits cell migration and invasion in vitro, but the possible molecular mechanism needs further research for deep understanding.

Emerging evidence revealed that cancer progression is tightly correlated with epithelial-to-mesenchymal transition (EMT), which induces the cancer cells to acquire mesenchymal phenotype and metastasize towards distant sites (30-32). Cancer cells could mislay the expression of cellular adhesion proteins (E-cadherin) and gain expression of mesenchymal markers ( $\mathrm{N}$-cadherin and vimentin) in the
EMT cascade $(33,34)$. The transcription factor zinc finger E-box-binding homeobox (ZEB) has an important role in initiation of EMT process. ZEB family factors (ZEB1 and ZEB2) were identified to act as transcriptional repressors that include two widely separated clusters of C2H2-type zinc fingers which bind to paired CAGGTA/G E-box-like promoter elements. These factors could trigger EMT through suppressing E-cadherin expression (35-37). EMT is modulated by a variety of signaling pathways and also by post-transcriptional mechanisms, including lncRNA (38). Therefore, to further investigate the molecular mechanism which knockdown of SNHG1 suppresses cell migration and invasion in vitro, the Saos-2 and U2OS cells were transfected with si-SNHG1 and si-Scramble, and the expression levels of ZEB1, E-cadherin, vimentin and N-cadherin were measured by western blot assays. Our results showed that knockdown of SNHG1 increased E-cadherin protein level but decreased ZEB1, vimentin and $\mathrm{N}$-cadherin protein levels in both Saos-2 and U2OS cells (Fig. 3C). Taken together, these results indicated that knockdown of SNHG1 suppresses cell migration and invasion via mediating EMT.

SNHG1 and miR-326 interact with and repress each other. Recently, IncRNA has been identified to be a sponge for modulating the expression and activity of miRNA $(21,22)$. To investigate if the expression of SNHG1 was regulated by miRNA, two bioinformatic databases including TargetScan 
A
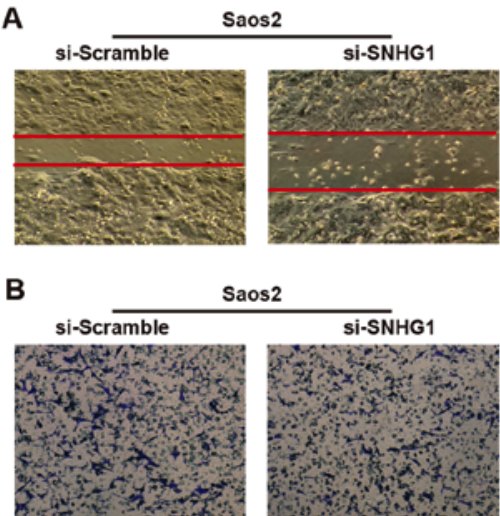

C

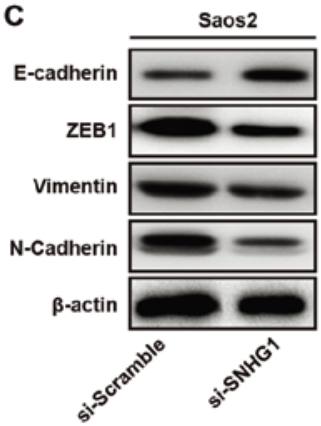

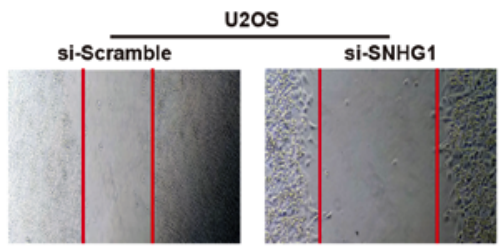

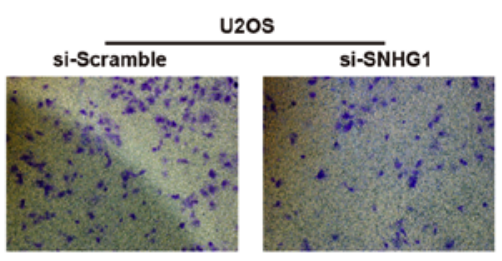

U2OS

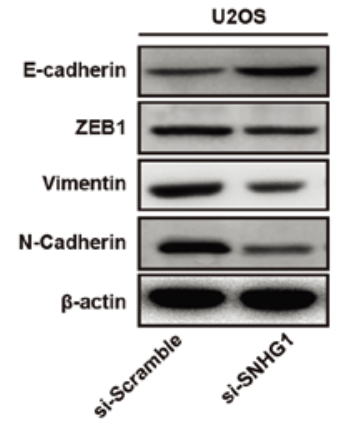

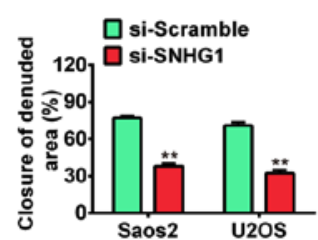
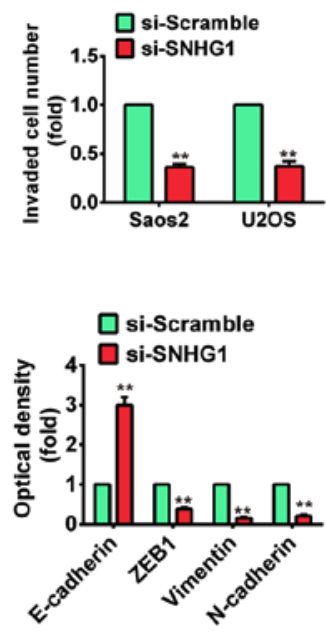

Figure 3. Knockdown of SNHG1 inhibits cell migration and invasion in vitro. (A and B) The effect of silencing SNHG1 expression on cell migration and invasion were monitored in SNHG1-downregulating Saos-2 and U2OS cells by wound healing and transwell assay, respectively. (C) The Saos-2 and U2OS cells were transfected with si-SNHG1 or si-Scramble and the expression of EMT marker genes E-cadherin, vimentin, N-cadherin and ZEB1 were evaluated using western blot analysis. $\beta$-actin was used as an internal control for protein loading. Data are presented as the mean \pm SD of three independent experiments. ${ }^{* *} \mathrm{P}<0.01$ vs. control.

A IncRNA-SNHG1
chr11:62620457-62620476
hsa-miR-326
IncRNA-SNHG1
chr11:62620457-62620557
hsa-miR-326

$$
\begin{aligned}
& 5^{\circ} \text { - ctctgtcctaGT CCCAGAG }-3 \\
& \text { 3' - gacetcettcCCGGGTCTCC - } 5 \\
& 5 \text { ' - tgaagttacaGTCCCAGAC }-3 \\
& \text { 3'- gacctccttcCCGGGTCTCC -5 } \\
& \text { Mut-SNHG1 GAACTCA }
\end{aligned}
$$
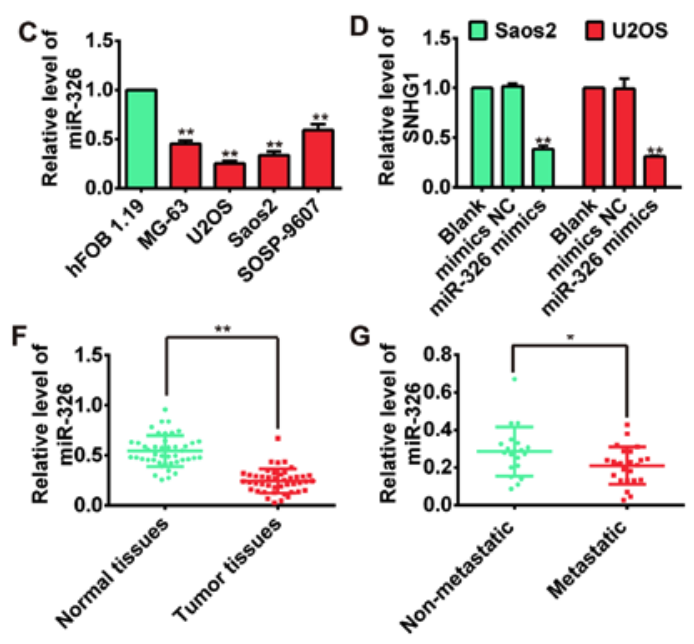
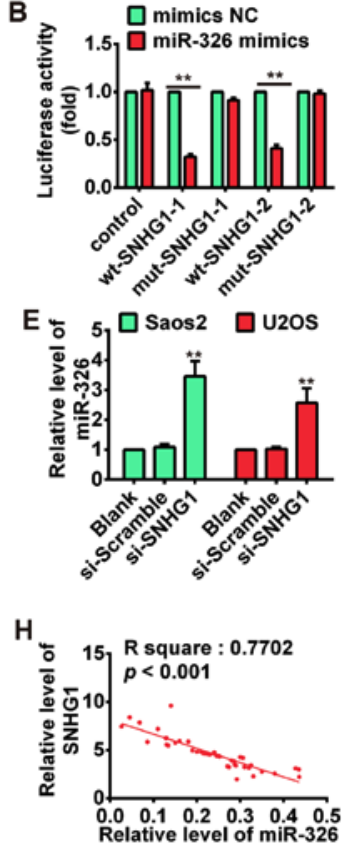

Figure 4. Reciprocal repression between SNHG1 and miR-326 in OS cells. (A) Diagram of the two miR-326 putative binding sites and corresponding mutant sites in SNHG1 sequences. (B) The relative luciferase activity of SNHG1 wild-type 1/2 or mutant 3'-UTR in Saos-2 cells following transfection with the miR-326 mimic or corresponding mimic NC. (C) The relative expressions of miR-326 were quantified using qRT-PCR in the four OS cell lines (MG-63, U2OS, Saos-2 and SOSP-9607) and human osteoblast cell line hFOB 1.19. (D) The qRT-PCR was used to detect SNHG1 level in Saos-2 and U2OS cells transfected with miR-326 mimics or mimics NC. (E) The relative expression of miR-326 was measured using qRT-PCR in Saos-2 and U2OS cells transfected with siSNHG1 or si-Scramble. (F) The relative expression of miR-326 in tumor tissues and normal tissues. (G) The relative expression of miR-326 in metastatic tissues and non-metastatic tissues. (H) The negative correlation between SNHG1 and miR-326 levels in the OS patients $\left(\mathrm{R}^{2}=0.7702, \mathrm{P}<0.001\right)$. Data are presented as the mean \pm SD of three independent experiments. ${ }^{*} \mathrm{P}<0.05 ;{ }^{* *} \mathrm{P}<0.01$ vs. control. 

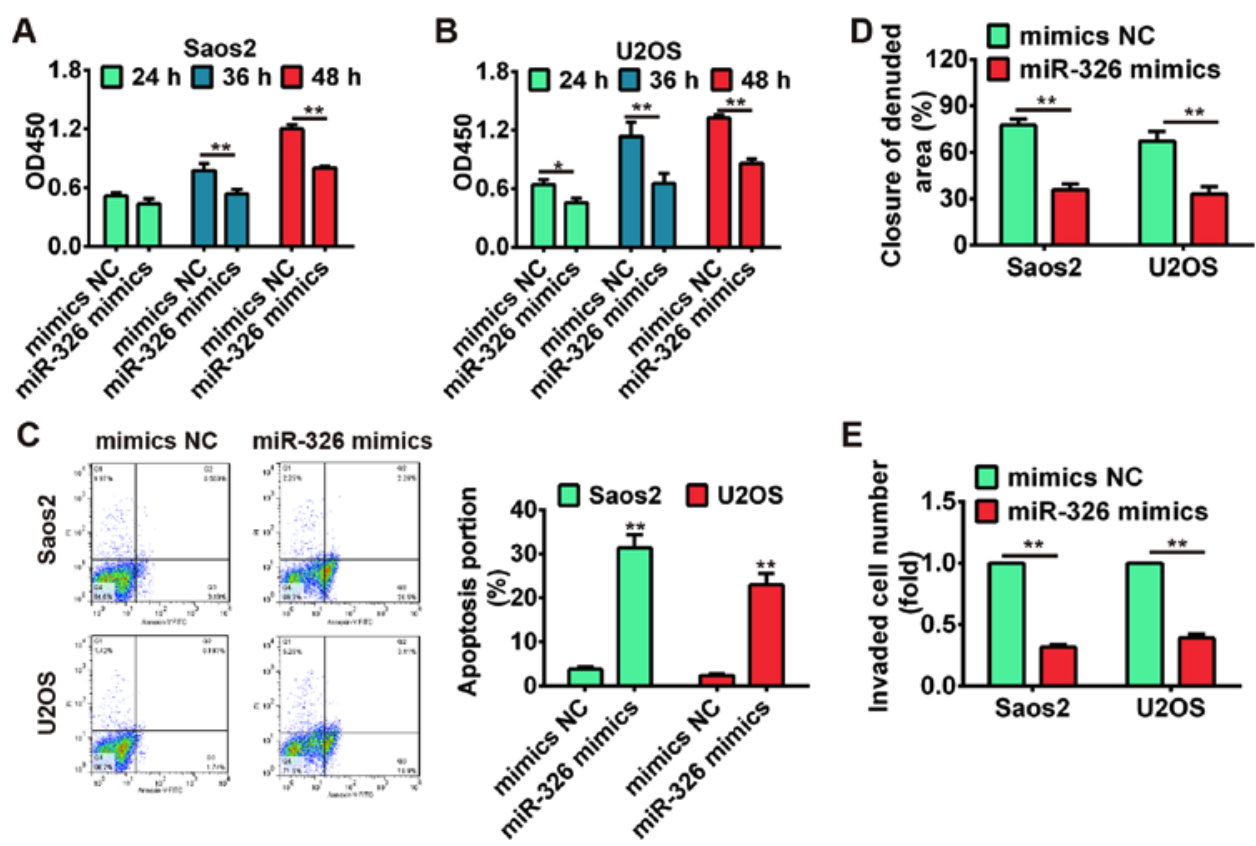

Figure 5. miR-326 inhibits cell proliferation, induces apoptosis and suppresses migration and invasion. (A and B) The Saos-2 and U2OS cells were transfected with miR-326 mimics or mimics NC and the CCK-8 assay was performed to detect the cell proliferation. (C) Flow cytometric analysis was performed to analyze cell apoptosis in miR-326 overexpressed Saos-2 and U2OS cells. (D and E) The effect of miR-326 overexpression on cell migration and invasion were monitored in Saos-2 and U2OS cells by wound healing and Transwell assay, respectively. Data are presented as the mean \pm SD of three independent experiments. ${ }^{* *} \mathrm{P}<0.01$ vs. control.

and microRNA.org were used to predict potential miRNAs, and found several potential miRNA-binding sites including miR-326. Previous studies documented that miR-326 may act as a tumor suppressor in a variety of cancers including glioma, medulloblastoma, cholangiocarcinoma and chronic lymphocytic leukemia (39-41). Our previous study also reported that miR-326 could repress the proliferation, migration and invasion of OS cells (42). Thus, the miR-326 was selected for further bioinformatic predication, we constructed the 3'-UTR reporter vectors coupled with full length of SNHG1 3'-UTR with wt or mut miR-326 binding site 1 or 2 (Fig. 4A). Luciferase assay demonstrated that miR-326 could suppress the expression of reporter gene containing wt 3'-UTR but not that containing mut 3'-UTR (Fig. 4B), suggesting SNHG1 is a direct target of miR-326. Moreover, we found that miR-326 is significantly upregulated in OS cell lines MG-63, U2OS, Saos-2, SOSP-9607 compared with osteoblastic cell line hFOB 1.19, exhibiting an inverse relationship with the SNHG1 expressions in OS cell lines ( $\mathrm{P}<0.01$; Fig. 4C). Furthermore, the results of qRT-PCR showed that the SNHG1 level could be repressed by miR-326 overexpression (Fig. 4D), whereas miR-326 expression was also upregulated after knockdown of SNHG1 in both Saos-2 and U2OS cells (Fig. 4E). Subsequently, we performed the qRT-PCR to determine the miR-326 level in tumor tissues. The result showed that the expression of miR-326 in tumor tissues was much lower than that in corresponding normal tissues $(\mathrm{P}<0.01$; Fig. 4F), whereas the miR-326 expression in metastatic tissues was also much lower than non-metastatic tissues ( $\mathrm{P}<0.01$; Fig. 4G). Moreover, correlation analysis presented a strong negative relationship between SNHG1 and miR-326 expression in OS tissues $\left(\mathrm{R}^{2}=0.7702, \mathrm{P}<0.001\right.$; Fig. $\left.4 \mathrm{H}\right)$. These data suggested that there was reciprocal repression between SNHG1 and miR-326.
miR-326 inhibits cell proliferation, induces apoptosis and suppresses migration and invasion. To further verify the tumor-suppressive effects of miR-326 on OS cells, the Saos-2 and U2OS cells were transfected with miR-326 mimics and mimics NC, and the cell proliferation, apoptosis, migration and invasion were investigated using CCK-8 assay, flow cytometric analysis, wound healing and Transwell invasion assay, respectively. Our results showed that overexpression of miR-326 significantly suppressed cell proliferation compared with mimics NC group ( $\mathrm{P}<0.01$; Fig. $5 \mathrm{~A}$ and $\mathrm{B})$, whereas miR-326 upregulation also induced apoptosis in both Saos-2 and U2OS cells (Fig. 5C). Additionally, the wound healing and transwell invasion assay demonstrated that cell migration and invasion were significantly inhibited after overexpression of miR-326 compared with mimics NC group $(\mathrm{P}<0.01$; Fig. 5D and E). These data indicated that miR-326 harbored the tumor-suppressive effects via suppressing cell growth, migration and invasion.

miR-326 suppresses NOB1 expression by directly targeting its 3 '-UTR. Increasing evidence revealed that the human nin one binding protein (NOB1) acts as an oncogene in different cancers (43-45), and miR-326 functions as a tumor suppressor by targeting the NOB1 in some cancers including colorectal carcinoma (46) and glioma (39). Therefore, we speculated whether NOB1 is a target of miR-326 in OS cells, and performed bioinformatic analysis to predict the putative targets of miR-326, and found that NOB1 might be a target gene of miR-326 and the target site located in the 3'-UTR (Fig. 6A). To verify this bioinformatic prediction, we constructed luciferase-reporter vectors containing the wt or mut 3'-UTR segments of NOB1 (Fig. 6A). The wt or mut reporter plasmid was cotransfected into Saos-2 cells along 


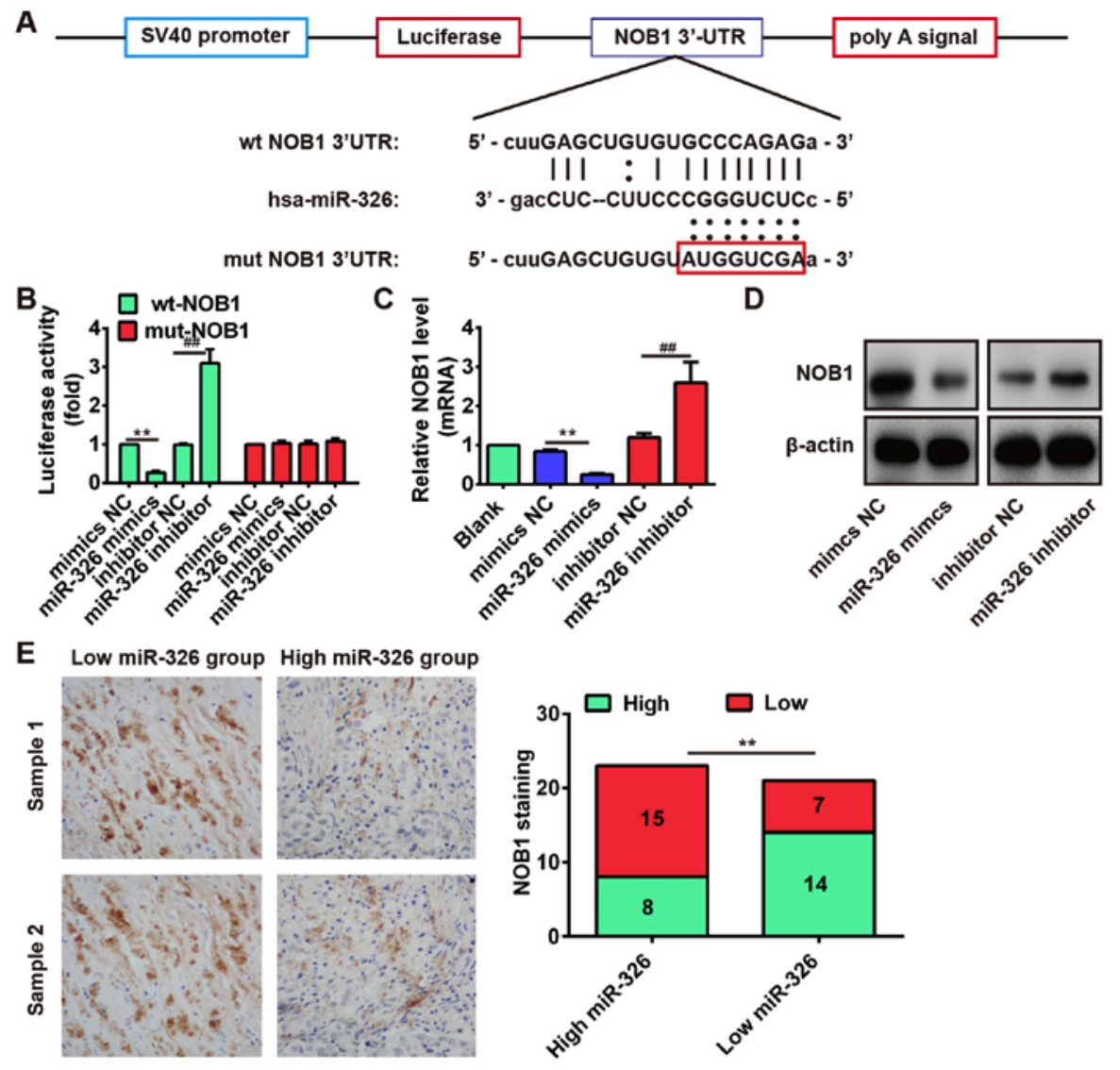

Figure 6. miR-326 suppresses NOB1 expression by directly targeting its 3'-UTR. (A) The PTEN 3'-UTR region containing the wt or mut binding site for miR-326. (B) The relative luciferase activity of NOB1 wt or mut 3'-UTR in Saos-2 cells after transfection with the miR-326 mimic/inhibitor or corresponding NC. ${ }^{* *} \mathrm{P}<0.01$ vs mimic NC. ${ }^{\# \#} \mathrm{P}<0.01$ vs. inhibitor NC. (C and D) The qRT-PCR and western blot analysis were performed to measure the mRNA and protein level of NOB1 in Saos-2 cells after transfection with the miR-326 mimic/inhibitor or corresponding NC, respectively. $\beta$-actin was used as an internal control for protein loading. ${ }^{* *} \mathrm{P}<0.01$ vs. mimic $\mathrm{NC}$. ${ }^{\# \#} \mathrm{P}<0.01$ vs. inhibitor NC. (E) Left panels: representative immunohistochemistry images showing the expression of NOB1 in OS tissues with low-expression or high-expression miR-326. Right panel: a significant inverse correlation between miR-326 and NOB1 expression in OS tissues $(\mathrm{r}=-0.5723, \mathrm{P}=0.0064)$. In the high miR-326 group $(\mathrm{n}=23)$, there were 15 cases with low expression of NOB1 and 8 cases with high expression of NOB1. In the low miR-326 group ( $\mathrm{n}=21$ ), there were 7 cases with low expression of NOB1 and 14 cases with high expression of NOB1. ${ }^{*} \mathrm{P}<0.01 \mathrm{vs}$. high miR-326 group. Data are presented as the mean $\pm \mathrm{SD}$ of three independent experiments.

with miR-326 mimics/inhibitor or NC, and measured the luciferase activity. We observed that miR-326 mimic significantly inhibited the luciferase activity compared with the mimic NC, but miR-326 inhibitor significantly enhanced the luciferase activity compared with the inhibitor $\mathrm{NC}$ in the presence of the wt 3'-UTR (P<0.01; Fig. 6B). Additionally, miR-326 did not repress the luciferase activity of the reporter vector containing 3'-UTR of NOB1 with mutations in the miR-326-binding site (Fig. 6B). To further determine that the expression of NOB1 is controlled by miR-326, we performed the western blot analysis and qRT-PCR analysis to detect the protein and mRNA level for NOB1, respectively. Our results showed that the protein and mRNA level of NOB1 were significantly inhibited after miR-326 overexpression, but markedly increased after knockdown of miR-326 compared with NC (Fig. 6C and D). These results demonstrated that NOB1 was a direct target for miR-326 in OS cells.

In addition, we performed the immunohistochemistry assay to measure the expression levels of NOB1 in OS tissues. Based on NOB1 expression level, the OS tissues were divided into low-expression group (scores 0 and 1) and high expression group (scores 2 and 3 ). In the high miR-326 group $(n=23)$, there were 15 cases with low expression of NOB1 and 8 cases with high expression of NOB1. In the low miR-326 group $(n=21)$, there were 7 cases with low expression of NOB1 and 14 cases with high expression of NOB1 (Fig. 6E). Furthermore, correlation analysis showed that the miR-326 expression was inversely correlated with NOB1 expression in OS tissues (Fig. 6E, $\mathrm{r}=-0.5723, \mathrm{P}=0.0064)$. These data indicated that NOB1 might be a target of miR-326 in OS tissues as well.

SNHG1 induces NOB1 upregulation via inhibiting miR-326 expression. Above results showed that SNHG1 could suppress the miR-326 which acts as a tumor suppressor by targeting the NOB1 in OS cells. Therefore, we speculated whether SNHG1 could indirectly mediate NOB1 expression via inhibiting the miR-326 level. To demonstrate this speculation, SNHG1 expression was dysregulated by SNHG1 overexpression plasmid pcDNA-SNHG1 or pcDNA-mut-SNHG1 transfection in Saos-2 cells, or cotransfection with miR-326 mimic, and the NOB1 expression was detected using western blot analysis. As shown in Fig. 7A, SNHG1 overexpression significantly increased NOB1 expression compared with blank, but SNHG1-induced upregulation of NOB1 was reversed by miR-326 overexpression in Saos-2 cells after co-transfection with miR-326 mimic and pcDNA-SNHG1. Furthermore, we 
A

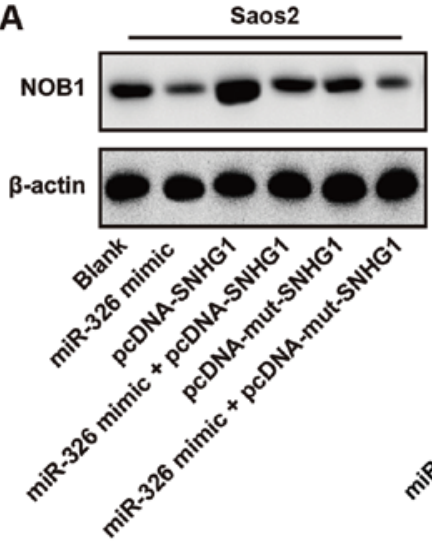

B

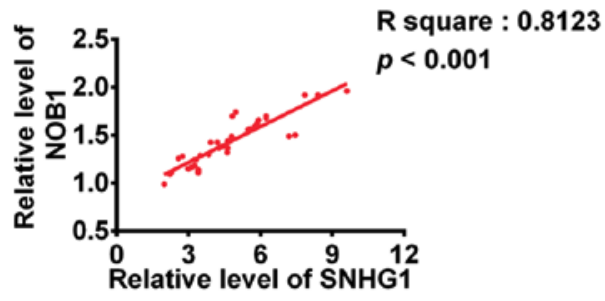

C
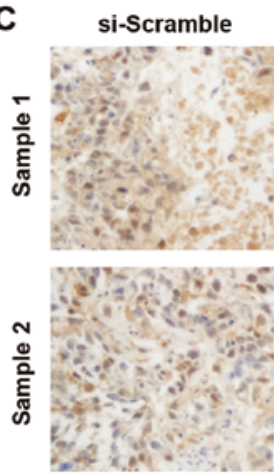

si-SNHG1

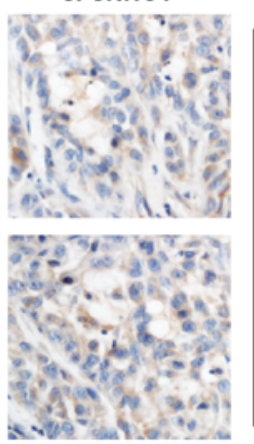

D

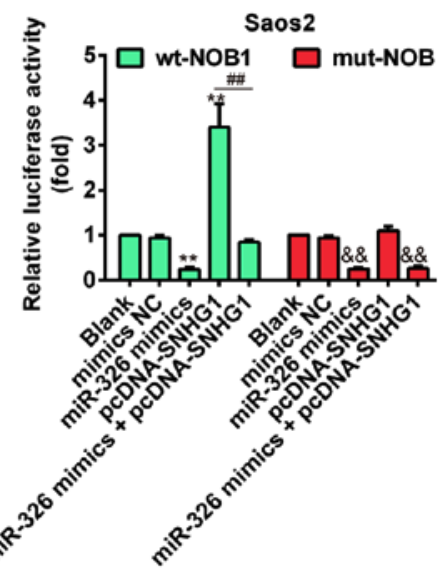

E

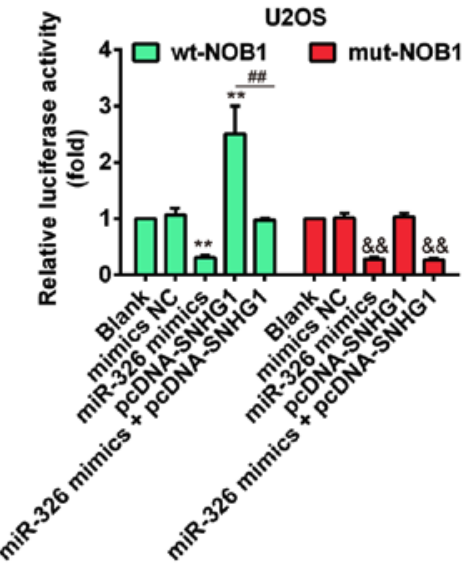

Figure 7. SNHG1 induces NOB1 upregulation via inhibiting miR-326 expression. (A) The Saos-2 cells were transfected with SNHG1 overexpression plasmid pcDNA-SNHG1 or pcDNA-mut-SNHG1, or cotransfected with miR-326 mimic and the NOB1 expression was detected using western blot analysis. ${ }^{* *} \mathrm{P}<0.01$ vs. blank. ${ }^{\# \#} \mathrm{P}<0.01$ vs. pcDNA-SNHG1. (B) The positive correlation between SNHG1 and NOB1 levels in the OS patients $\left(\mathrm{R}^{2}=0.8123\right.$, $\left.\mathrm{P}<0.001\right)$. (C) The immunohistochemistry assay was used to detect the NOB1 expression level in OS mouse model injected with SNHG1-downregulating (si-SNHG1) Saos-2 or parallel si-Scramble carrying cells. (D and E) The relative luciferase activity of NOB1 wt or mut 3'-UTR in Saos-2 and U2OS cells after transfection with miR-326 mimics, pcDNA-SNHG1, mimics NC or miR-326 mimics + pcDNA-SNHG1. ${ }^{* *} \mathrm{P}<0.01$ vs. Blank, ${ }^{\# \#} \mathrm{P}<0.01$ vs. pcDNA-SNHG1 group in NOB1 wt groups. ${ }^{\& \&} \mathrm{P}<0.01$ vs. Blank in NOB1 mut groups.

observed that mut-SNHG1 overexpression did not affect the NOB1 level, whereas miR-326 upregulation also significantly reduced NOB1 expression in Saos-2 cells after co-transfection with miR-326 mimic and pcDNA-mut-SNHG1 compared with blank $(\mathrm{P}<0.01$; Fig. 7A). Additionally, correlation analysis further verified a strong positive relationship between SNHG1 and NOB1 expression in OS tissues $\left(\mathrm{R}^{2}=0.8123\right.$, $\mathrm{P}<0.01$; Fig. 7B). Furthermore, immunohistochemical results also demonstrated that knockdown of SNHG1 increased the NOB1 expression level in xenograft tumor tissues (Fig. 7C). These results suggested that SNHG1 may indirectly increase NOB1 level through regulating miR-326 expression. To further explore this mechanism, the wt or mut reporter plasmid that contained the wt or mut 3'-UTR segments of NOB1 was cotransfected into Saos-2 and U2OS cells along with miR-326 mimics, pcDNA-SNHG1, mimics NC, or miR-326 mimics + pcDNA-SNHG1 and measured the luciferase activity. Our results showed that miR-326 mimics or pcDNA-SNHG1 significantly inhibited or enhanced the luciferase activity compared with the NC (blank and mimic NC) in the presence of the wt 3'-UTR, but the pcDNA-SNHG1-enhanced effect was abolished by miR-326 mimics in Saos- 2 and U2OS cells after cotransfection with miR-326 mimics and pcDNASNHG1 (Fig. 7D and E). In addition, pcDNA-SNHG1 did not enhance the luciferase activity compared with the NC (blank and mimic NC) in the presence of the mut 3'-UTR. These data indicated that the tumorigenic SNHG1 upregulated NOB1 expression via suppressing miR-326 level in OS.

\section{Discussion}

Accumulating evidence demonstrated that ectopic expression of lncRNAs could act as oncogenes or tumor suppressors in various cancer progression and development (13). However, only few reports have discussed their contribution in OS. In the present study, we found that SNHG1 was upregulated in OS tissues and cell lines. Knockdown of SNHG1 inhibited cell proliferation, migration and invasion, and induced apoptosis via activating cleaved-caspase-3. Meanwhile, the xenograft model showed that SNHG1 downregulation suppressed tumor 
growth in vivo. Furthermore, our data confirmed that there was reciprocal repression between SNHG1 and miR-326 which functioned as a tumor suppressor through suppressing NOB1 expression. Additionally, we verified that tumorigenic SNHG1 modulated NOB1 expression via suppressing miR-326 level in OS, suggesting that SNHG1 might function as an oncogene in OS through upregulating NOB1 level.

Many studies confirmed that SNHG1 was upregulated in different cancers, such as lung, breast cancer and hepatocellular carcinoma (24-26). SNHG1 overexpression is associated with poor prognosis of hepatocellular carcinoma patients (24). However, the potential roles of SNHG1 in OS remain unclear. Consistent with previous studies, our results also showed that SNHG1 was upregulated in OS tissues and cell lines, and Kaplan-Meier survival analysis demonstrated that high SNHG1 expression predicts poor overall survival of OS patients. These data suggested that SNHG1 was dysregulated in OS, and might function as an oncogene in OS tumorigenesis. To further explore this mechanism, the SNHG1 function was investigated using siRNA-mediated knockdown experiments. We observed that knockdown of SNHG1 suppressed cell proliferation, migration and invasion and prompted apoptosis via activating cleaved-caspase-3 pathway. Xenograft tumor model showed that knockdown of SNHG1 inhibited tumor growth in vivo. Increasing evidence identified that cancer is tightly involved with EMT, which induces the cancer cells to acquire mesenchymal phenotype and metastasize towards distant sites (30-32). Therefore, to further explore the molecular mechanism which knockdown of SNHG1 suppresses cell migration and invasion in vitro, we performed western blot analysis to determine EMT markers (E-cadherin, vimentin, $\mathrm{N}$-cadherin and ZEB1) in OS cells after SNHG1 downregulation. Our data showed that knockdown of SNHG1 increased E-cadherin protein level but decreased vimentin, $\mathrm{N}$-cadherin and ZEB1 which promoted EMT by repressing expression of E-cadherin (35-37). These results suggested that knockdown of SNHG1 suppresses cell migration and invasion via modulating EMT.

In recent years, ceRNA hypothesis has been widely reported and some studies have verified the interaction between IncRNA and miRNA in cancers $(47,48)$, we hypothesized that SNHG1 may act as a ceRNA in OS. To confirm our hypothesis, we used two bioinformatic databases including TargetScan and microRNA.org to predict potential miRNAs, and found several potential miRNA-binding sites including miR-326. Recent studies, and our previous demonstration, indicated that miR-326 may act as a tumor suppressor in many cancers including OS (39-41). Thus, the miR-326 was selected and further investigated by bioinformatics. Our results showed that SNHG1 is a direct target of miR-326, and overexpression of miR-326 abolished SNHG1 upregulation in OS cells, conversely, SNHG1 downregulation increased miR-326 expression levels. Furthermore, we found that miR-326 was downregulated in OS tissues especially metastatic tissues and cell lines, and exhibited an inverse correlation with SNHG1 expression in OS tissues. These results suggested that the reciprocal regulation of SNHG1 and miR-326 might have an important role in OS progression.

Increasing evidence identified that miR-326 functions as a tumor suppressor by targeting the NOB1 which acts as an oncogene in different cancers $(39,43-46)$. Consistent with previous studies, our data further confirmed that miR-326 repressed cell proliferation, migration and invasion, and induced apoptosis via directly targeting NOB1 3'-UTR. Moreover, miR-326 expression was inversely correlated with NOB1 expression in OS tissues. Based on these results, we speculated whether SNHG1 could indirectly modulate NOB1 expression as ceRNA to inhibit the activity of miR-326. Our results showed that upregulation of SNHG1 increased NOB1 expression, but SNHG1-induced upregulation of NOB1 was reversed by miR-326 overexpression. Furthermore, further experiments verified a strong negative relationship between SNHG1 and miR-326 expression in OS tissues, and the miR-326 suppressive effect on NOB1 was abolished by SNHG1 overexpression in OS cells. Taken together, these results indicated tumorigenic SNHG1 as a sponge to suppress miR-326 activity by sequestering it away from its target NOB1 in OS.

Recently, IncRNAs have been demonstrated to be involved in differentiation, proliferation and development, as well as cell cycle regulation and programmed cell death $(49,50)$. Previous studies reported that lncRNAs were identified to act as oncogenes or tumor suppressor genes to regulate OS progression, such as H19, MALAT1, ANCR, HOTAIR, TUG1, MEG3, HIF2PUT and TUSC7 (16). According to the ceRNA hypothesis, lncRNAs interacted with other RNA transcripts via miRNA response elements as the letters of a novel RNA language (6). Liang et al (51) revealed that H19 promotes epithelial to mesenchymal transition and acts as a ceRNA for miR-138 and miR-200a in colorectal cancer. Therefore, we speculated that more IncRNAs may be involved in the progression of OS, whether SNHG1 functions as a ceRNA for more miRNAs need to be further investigated in subsequent studies.

In summary, our results demonstrated that SNHG1 was upregulated in OS tissues and cell lines. Knockdown of SNHG1 suppressed OS cell growth and metastasis in vitro and in vivo. More importantly, we verified that SNHG1 inhibits the activity of miR-326 by acting as an endogenous sponge, through increasing the expression of its target gene, NOB1. The present study not only uncovered the important role of SNHG1/miR-326/NOB1 signaling pathway in OS pathogenesis but also implicated the potential role of both SNHG1 and miR-326 in the OS clinical diagnosis and treatment.

\section{Acknowledgemens}

The present study was supported by the National Natural Science Foundation of China (no. 81301588).

\section{References}

1. Link MP, Goorin AM, Miser AW, Green AA, Pratt CB, Belasco JB, Pritchard J, Malpas JS, Baker AR, Kirkpatrick JA, et al: The effect of adjuvant chemotherapy on relapse-free survival in patients with osteosarcoma of the extremity. N Engl J Med 314: 1600-1606, 1986.

2. Mirabello L, Troisi RJ and Savage SA: Osteosarcoma incidence and survival rates from 1973 to 2004: Data from the Surveillance, Epidemiology, and End Results Program. Cancer 115: 1531-1543, 2009.

3. Marina N, Gebhardt M, Teot L and Gorlick R: Biology and therapeutic advances for pediatric osteosarcoma. Oncologist 9: $422-441,2004$ 
4. Song QC, Shi ZB, Zhang YT, Ji L, Wang KZ, Duan DP and Dang XQ: Downregulation of microRNA-26a is associated with metastatic potential and the poor prognosis of osteosarcoma patients. Oncol Rep 31: 1263-1270, 2014.

5. Cho WCS: MicroRNAs: Potential biomarkers for cancer diagnosis, prognosis and targets for therapy. Int J Biochem Cell Biol 42: 1273-1281, 2010

6. Khoury S: Circulating microRNAs : potential biomarkers for common malignancies. Biomark Med 9: 131-151, 2015.

7. Liang WC, Wang Y, Xiao LJ, Wang YB, Fu WM, Wang WM, Jiang HQ, Qi W, Wan DC, Zhang JF, et al: Identification of miRNAs that specifically target tumor suppressive KLF6-FL rather than oncogenic KLF6-SV1 isoform. RNA Biol 11: $845-854,2014$

8. Calin GA and Croce CM: MicroRNA signatures in human cancers. Nat Rev Cancer 6: 857-866, 2006.

9. Lipovich L, Johnson R and Lin CY: MacroRNA underdogs in a microRNA world: Evolutionary, regulatory, and biomedical significance of mammalian long non-protein-coding RNA. Biochim Biophys Acta 1799: 597-615, 2010.

10. Peng W and Fan H: Long non-coding RNA PANDAR correlates with poor prognosis and promotes tumorigenesis in hepatocellular carcinoma. Biomed Pharmacother 72: 113-118, 2015.

11. Deng J, Liang Y, Liu C, He S and Wang S: The up-regulation of long non-coding RNA AFAP1-AS1 is associated with the poor prognosis of NSCLC patients. Biomed Pharmacother 75: 8-11, 2015.

12. Gibb EA, Brown CJ and Lam WL: The functional role of long non-coding RNA in human carcinomas. Mol Cancer 10: 38-38, 2011.

13. Prensner JR and Chinnaiyan AM: The emergence of lncRNAs in cancer biology. Cancer Discov 1: 391-407, 2011.

14. Li L, Zhang L, Zhang Y and Zhou F: Increased expression of LncRNA BANCR is associated with clinical progression and poor prognosis in gastric cancer. Biomed Pharmacother 72 : $109-112,2015$

15. Chen J, Wang Y and Wang D: Noise perturbation improves supervised speech separation. In: Latent Variable Analysis and Signal Separation: Proc of 12th International Conference, LVA/ ICA 2015, Liberec, Czech Rep, August 25-28, 2015. Vincent E, Yeredor A, Koldovský Z and Tichavský P (eds.) Springer International Publishing, Cham, pp83-90, 2015.

16. Yang Z, Li X, Yang Y, He Z, Qu X and Zhang Y: Long noncoding RNAs in the progression, metastasis, and prognosis of osteosarcoma. Cell Death Dis 7: e2389, 2016.

17. Cong M, Li J, Jing R and Li Z: Long non-coding RNA tumor suppressor candidate 7 functions as a tumor suppressor and inhibits proliferation in osteosarcoma. Tumour Biol 37 9441-9450, 2016.

18. Li F, Cao L, Hang D, Wang F and Wang Q: Long non-coding RNA HOTTIP is up-regulated and associated with poor prognosis in patients with osteosarcoma. Int J Clin Exp Pathol 8: 11414-11420, 2015.

19. Zhuang LK, Xu GP, Pan XR, Lou YJ, Zou QP, Xia D, Yan WW, Zhang YT, Jia PM and Tong JH: MicroRNA-181a-mediated downregulation of AC9 protein decreases intracellular cAMP level and inhibits ATRA-induced APL cell differentiation. Cell Death Dis 5: e1161, 2014

20. Zhang B, Pan X, Cobb GP and Anderson TA: microRNAs as oncogenes and tumor suppressors. Dev Biol 302: 1-12, 2007.

21. Ma MZ, Chu BF, Zhang Y, Weng MZ, Qin YY, Gong W and Quan ZW: Long non-coding RNA CCAT1 promotes gallbladder cancer development via negative modulation of miRNA-218-5p Cell Death Dis 6: e1583, 2015.

22. Cui M, Xiao Z, Wang Y, Zheng M, Song T, Cai X, Sun B, Ye L and Zhang X: Long noncoding RNA HULC modulates abnormal lipid metabolism in hepatoma cells through an miR-9-mediated RXRA signaling pathway. Cancer Res 75: 846-857, 2015.

23. Salmena L, Poliseno L, Tay Y, Kats L and Pandolfi PP: A ceRNA hypothesis: The Rosetta Stone of a hidden RNA language? Cell 146: 353-358, 2011

24. Zhang M, Wang W, Li T, Yu X, Zhu Y, Ding F, Li D and Yang T: Long noncoding RNA SNHG1 predicts a poor prognosis and promotes hepatocellular carcinoma tumorigenesis. Biomed Pharmacother 80: 73-79, 2016.

25. You J, Fang N, Gu J, Zhang Y, Li X, Zu L and Zhou Q: Noncoding RNA small nucleolar RNA host gene 1 promote cell proliferation in nonsmall cell lung cancer. Indian J Cancer 51: e99-e102, 2014
26. Yu F, Bracken CP, Pillman KA, Lawrence DM, Goodall GJ, Callen DF and Neilsen PM: p53 represses the oncogenic Sno-MiR-28 derived from a SnoRNA. PLoS One 10: e0129190, 2015.

27. Li J, Zhang Z, Xiong L, Guo C, Jiang T, Zeng L, Li G and Wang J: SNHG1 lncRNA negatively regulates miR-199a-3p to enhance CDK7 expression and promote cell proliferation in prostate cancer. Biochem Biophys Res Commun 487: 146-152, 2017.

28. Zhang H, Zhou D, Ying M, Chen M, Chen P, Chen Z and Zhang F: Expression of long non-coding RNA (lncRNA) small nucleolar RNA host gene 1 (SNHG1) exacerbates hepatocellular carcinoma through suppressing miR-195. Med Sci Monit 22: 4820-4829, 2016.

29. Zhou L, Rui JA, Ye DX, Wang SB, Chen SG and Qu Q: Edmondson-Steiner grading increases the predictive efficiency of TNM staging for long-term survival of patients with hepatocellular carcinoma after curative resection. World J Surg 32: 1748-1756, 2008.

30. Hollier BG, Evans K and Mani SA: The epithelial-to-mesenchymal transition and cancer stem cells: A coalition against cancer therapies. J Mammary Gland Biol Neoplasia 14: 29-43, 2009.

31. Ahmed N, Abubaker K, Findlay J and Quinn M: Epithelial mesenchymal transition and cancer stem cell-like phenotypes facilitate chemoresistance in recurrent ovarian cancer. Curr Cancer Drug Targets 10: 268-278, 2010.

32. Latifi A, Abubaker K, Castrechini N, Ward AC, Liongue C, Dobill F, Kumar J, Thompson EW, Quinn MA, Findlay JK, et al: Cisplatin treatment of primary and metastatic epithelial ovarian carcinomas generates residual cells with mesenchymal stem cell-like profile. J Cell Biochem 112: 2850-2864, 2011.

33. Hur K, Toiyama Y, Takahashi M, Balaguer F, Nagasaka T, Koike J, Hemmi H, Koi M, Boland CR and Goel A: MicroRNA$200 \mathrm{c}$ modulates epithelial-to-mesenchymal transition (EMT) in human colorectal cancer metastasis. Gut 62: 1315-1326, 2013.

34. Cesi V,Casciati A, Sesti F, Tanno B, Calabretta B and Raschellà G: TGF $\beta$-induced c-Myb affects the expression of EMT-associated genes and promotes invasion of $\mathrm{ER}^{+}$breast cancer cells. Cell Cycle 10: 4149-4161, 2011.

35. Derynck R and Akhurst RJ: Differentiation plasticity regulated by TGF-beta family proteins in development and disease. Nat Cell Biol 9: 1000-1004, 2007.

36. Kalluri R and Weinberg RA: The basics of epithelial-mesenchymal transition. J Clin Invest 119: 1420-1428, 2009.

37. Vandewalle C, Van Roy F and Berx G: The role of the ZEB family of transcription factors in development and disease. Cell Mol Life Sci 66: 773-787, 2009.

38. Ma C, Nong K, Zhu H, Wang W, Huang X, Yuan Z and Ai K: H19 promotes pancreatic cancer metastasis by derepressing let-7's suppression on its target HMGA2-mediated EMT. Tumour Biol 35: 9163-9169, 2014.

39. Zhou J, Xu T, Yan Y, Qin R, Wang H, Zhang X, Huang Y, Wang Y, Lu Y, Fu D, et al: MicroRNA-326 functions as a tumor suppressor in glioma by targeting the Nin one binding protein (NOB1). PLoS One 8: e68469, 2013.

40. Meng F, Henson R, Lang M, Wehbe H, Maheshwari S, Mendell JT, Jiang J, Schmittgen TD and Patel T: Involvement of human micro-RNA in growth and response to chemotherapy in human cholangiocarcinoma cell lines. Gastroenterology 130: 2113-2129, 2006

41. Ferretti E, De Smaele E, Miele E, Laneve P, Po A, Pelloni M, Paganelli A, Di Marcotullio L, Caffarelli E, Screpanti I, et al: Concerted microRNA control of Hedgehog signalling in cerebellar neuronal progenitor and tumour cells. EMBO J 27: 2616-2627, 2008

42. Cao L, Wang J and Wang PQ: MiR-326 is a diagnostic biomarker and regulates cell survival and apoptosis by targeting Bcl-2 in osteosarcoma. Biomed Pharmacother 84: 828-835, 2016.

43. He XW, Feng T, Yin QL, Jian YW and Liu T: NOB1 is essential for the survival of RKO colorectal cancer cells. World J Gastroenterol 21: 868-877, 2015.

44. Liu K, Chen HL, Gu MM and You QS: NOB1 expression predicts early response to cisplatin-based chemotherapy in patients with advanced non-small cell lung cancer. J Chemother 28: 225-229, 2016.

45. Huang P, Xi J and Liu S: MiR-139-3p induces cell apoptosis and inhibits metastasis of cervical cancer by targeting NOB1. Biomed Pharmacother 83: 850-856, 2016. 
46. Wu L, Hui H, Wang LJ, Wang $\mathrm{H}$, Liu QF and Han SX MicroRNA-326 functions as a tumor suppressor in colorectal cancer by targeting the nin one binding protein. Oncol Rep 33 2309-2318, 2015

47. Zhang Z, Zhu Z, Watabe K, Zhang X, Bai C, Xu M, Wu F and Mo YY: Negative regulation of lncRNA GAS5 by miR-21. Cell Death Differ 20: 1558-1568, 2013.

48. Liu XH, Sun M, Nie FQ, Ge YB, Zhang EB, Yin DD, Kong R, Xia R, Lu KH, Li JH, et al: Lnc RNA HOTAIR functions as a competing endogenous RNA to regulate HER 2 expression by sponging miR-331-3p in gastric cancer. Mol Cancer 13: 92, 2014

49. Wu L, Jin L, Zhang W and Zhang L: Roles of long non-coding RNA CCAT2 in cervical cancer cell growth and apoptosis. Med Sci Monit 22: 875-879, 2016.
50. Ballantyne MD, Pinel K, Dakin R, Vesey AT, Diver L, Mackenzie R, Garcia R, Welsh P, Sattar N, Hamilton G, et al: Smooth muscle enriched long noncoding RNA (SMILR) regulates cell proliferation. Circulation 133: 2050-2065, 2016

51. Liang WC, Fu WM, Wong CW, Wang Y, Wang WM, Hu GX, Zhang L, Xiao LJ, Wan DC, Zhang JF, et al: The lncRNA H19 promotes epithelial to mesenchymal transition by functioning as miRNA sponges in colorectal cancer. Oncotarget 6: 22513-22525, 2015.

This work is licensed under a Creative Commons Attribution-NonCommercial-NoDerivatives 4.0 International (CC BY-NC-ND 4.0) License. 